\title{
Heart Protection by Combination Therapy with Esmolol and Milrinone at Late-Ischemia and Early Reperfusion
}

\author{
Ming-He Huang • Yewen Wu • Vincent Nguyen • Saurabh Rastogi • \\ Bradley K. McConnell • Cori Wijaya • Barry F. Uretsky • Kian-Keong Poh • \\ Huay-Cheem Tan • Kenichi Fujise
}

Published online: 12 May 2011

(C) The Author(s) 2011. This article is published with open access at SpringerLink.com

\begin{abstract}
Introduction The present study determined whether lateischemia/early reperfusion therapy with the $\beta_{1}$-adrenergic receptor (AR) blocker esmolol and phosphodiesterase III inhibitor milrinone reduced left ventricular (LV) myocardial infarct size (IS).

Methods and Results In an ischemia/reperfusion rat model (30-min ischemia/4-hr reperfusion), esmolol, milrinone or esmolol + milrinone were intravenous (IV) infused over $10 \mathrm{~min}$ (from the last $5 \mathrm{~min}$ of ischemia to the first $5 \mathrm{~min}$ of reperfusion). LV-IS were $48.9 \pm 8.9 \%, 41.5 \pm 5.4 \%, 25.8 \pm$ $7.7 \%$ and $16.8 \pm 7.3 \%$ for saline, esmolol, milrinone, and esmolol + milrinone, respectively ( $n=12 /$ group). Esmolol + milrinone further reduced LV-IS compared with esmolol or milrinone alone $(p<0.05)$. LV-IS-reduction induced by
\end{abstract}

M.-H. Huang $\cdot$ Y. Wu $\cdot$ V. Nguyen $\cdot$ S. Rastogi $\cdot$ B. F. Uretsky $\cdot$ K. Fujise

Department of Internal Medicine, Cardiology Division (M-HH, YW, VN, SR, BFU, KF), University of Texas Medical Branch, Galveston, TX, USA

M.-H. Huang ( $\square)$

Gulf Coast Heart Clinic (M-HH), League City,

2785 Gulf Freeway S, Suite 155,

League, TX 77573, USA

e-mail: mhuang1458@aol.com

M.-H. Huang

e-mail: mihuang@utmb.edu

B. K. McConnell • C. Wijaya

Department of Pharmacological and Pharmaceutical Sciences,

College of Pharmacy (BKM, CW), University of Houston,

Houston, TX, USA

K.-K. Poh $\cdot$ H.-C. Tan

Cardiac Department (K-KP, H-CT), National University Hospital,

Singapore, Singapore esmolol + milrinone was eliminated in the presence of protein kinase A-(PKA)-inhibitor (Rp-cAMPS) or Aktinhibitor (AKT 1/2 kinase inhibitor). In mixed rat ventricular cardiomyocyte cultures, intra-ischemic application of esmolol, milrinone or esmolol + milrinone reduced myocyte death rates by $5.5 \%, 13.3 \%$, and $16.8 \%$, respectively, compared with saline $(p<0.01)$. This cell protective effect by esmolol + milrinone was abrogated in the presence of PKA-inhibitor or Akt-inhibitor. Esmolol, milrinone or esmolol + milrinone increased myocardial PKA activity by $22 \%, 28 \%$ and $59 \%$, respectively, compared with saline $(n=6, p<0.01)$. No nonspecific adverse effect of Rp-cAMPS on myocytes was identified in a purified myocyte preparation during hypoxia/ re-oxygenation. Antiapoptotic pathways were assessed by measuring myocardial phosphorylated Akt (pAkt) levels combined with terminal dUTP nick-end labelling staining analysis. Ten minutes following infusion of esmolol, milrinone or esmolol + milrinone, there were 1.7-, 2.7-, and 6-fold increase in tissue pAkt levels, respectively. This esmolol + milrinone induced pAkt activation was abolished in the presence of PKA inhibitor. Esmolol, milrinone and esmolol + milrinone reduced myocyte apoptosis rates by $22 \%, 37 \%$ and $60 \%$, respectively, compared with saline $(p<0.01)$.

Conclusions Late-ischemia/early reperfusion therapy with esmolol+milrinone additively reduces LV-IS associated with robust activation of myocardial PKA and subsequent Akt-antiapoptotic pathway.

Key words Akt $\cdot$ Esmolol $\cdot$ Milrinone $\cdot$ PKA $\cdot$ Reperfusion injury

\section{Introduction}

While the translation of ischemic preconditioning to clinical application remains unrealistic for patients with acute 
myocardial infarction (AMI), recent research in ischemic postconditioning opens new opportunity for potentially accessible clinical treatment for AMI. Reperfusion injury salvage kinase (RISK) pathway has been implicated in the postconditioning for infarct-size (IS) reduction [1, 2]. Among several potential mechanisms, Akt activation has been implicated as one of the key signaling pathways underling postconditioning mediated heart protection [1,2], although species difference has been reported [3]. The mammalian heart possesses adrenopeptidergic neuroparacrine system consisting of intrinsic cardiac adrenergic (ICA) cells [4-6]. ICA cells synthesize and release epinephrine and calcitonin gene-related peptide (CGRP), stimulating myocardial $\beta_{2}$-adrenoreceptor $\left(\beta_{2}\right.$-AR) and CGRP-receptors $[5,6]$, both of which being coupled to the adenylate cyclase-cAMP-dependent signaling pathway [7-9]. Concomitant stimulation of cardiac $\beta_{2}$-AR/CGRPreceptors before ischemia or at the beginning of the reperfusion confers robust IS-reduction [10]. This adrenopeptidergic signaling is mediated through the activation of cAMP-dependent PKA [10] which, in turn, activates Akt, a powerful antiapoptotic signaling pathway $[1,2,11,12]$. It is conceivable that medications sharing similar distal signaling mechanisms of $\beta_{2}$-AR/CGRP-receptors may provide alternatives for the receptor-mediated cardioprotection. For instance, direct myocardial PKA activation with a phosphodiesterase (PDE) III inhibitor milrinone at reperfusion may confer equivalent cardioprotection to that conferred by $\beta_{2}$-AR/CGRP-receptor stimulation, since milrinone is known to activate PKA $[13,14]$. High dose of $\beta_{1}$-AR blocker esmolol given at the onset of reperfusion reduces LV-IS [15]. This cardioprotective effect is likely mediated, in part, through the enhanced $\beta_{2}$-AR signaling promoting myocyte survival [11, 12], since at early reperfusion, there is $>100$ fold increase in myocardial interstitial epinephrine release [16] exerting unopposed myocardial $\beta_{2}$-AR stimulation in the presence of $\beta_{1}$-AR blockade. Although, it has been reported that intra-ischemic PKA activation with milrinone reduces right ventricular IS in dogs [17], it has not been demonstrated whether intra-ischemic/reperfusion therapy with milrinone reduces LV-IS. Furthermore, the mechanism underlying post-ischemia milrinone-induced heart protection has not been elucidated. We have hypothesized that cardiac PKA activation with the selective $\beta_{1}$-AR blocker esmolol in combination with milrinone at lateischemia/early reperfusion decreases LV-IS. The present study had the following objectives: to determine if: 1) LVIS can be additively reduced by cardiac PKA activation with combination therapy of esmolol + milrinone delivered at the beginning of reperfusion, in a manner dependent of PKA and Akt activation; 2) myocyte death rates can be reduced by intra-ischemic treatment with esmolol + milrinone in vitro, 3) combination therapy with esmolol + milrinone additively enhance myocardial PKA and PKAdependent Akt activation; and 4) esmolol + milrinone-induced Akt activation results in reduced myocyte apoptosis during ischemia/reperfusion.

\section{Materials and methods}

Effect of esmolol + milrinone on LV-IS-reduction The rat AMI model consisted of 30-min ischemia and 4-hr reperfusion $[5,6,18]$. Protocols for animal studies were approved by the Institutional Animal Care and the Use Committee of the University of Texas Medical Branch. Saline, esmolol $(10 \mu \mathrm{g} / \mathrm{kg} / \mathrm{min})$, milrinone $(5 \mu \mathrm{g} / \mathrm{kg} / \mathrm{min})$ or esmolol + milrinone were infused via the tail veins over 10-min (5-min before and after the onset of reperfusion, $n=$ 12/group). Arterial blood pressure (BP) and heart rate (HR) were monitored via an artery catheter (iWorx/CB Sciences, Inc, Dover, NH). It should be noted that to avoid significant hemodynamic changes, the milrinone dose applied in the present study is only $1 / 10$ of that recommended loading dose for clinical heart failure treatment and esmolol dose is $1 / 5$ of that maintenance dose for the treatment of clinical supraventricular tachycardia. To test whether esmolol + milrinone induced effect was dependent on PKA activation, LV-IS was assessed in the animals $(n=12)$ who received PKA inhibitor Rp-cAMPS $(2 \mathrm{mg} / \mathrm{kg}$, infused at $10 \mathrm{~min}$ after coronary artery occlusion) followed by infusion of esmolol + milrinone. The effect of Rp-cAMPS alone on IS was tested in 8 animals. To determine whether esmolol's effect was mediated through the enhanced endogenous cardiac $\beta_{2}$-AR stimulation, the effect of esmolol on IS was tested in the animals $(n=8)$ pretreated with the selective $\beta_{2}$-AR antagonist ICI-118,551 $(200 \mu \mathrm{g} / \mathrm{kg} / \mathrm{min}$ for $5 \mathrm{~min})$ infused at $10 \mathrm{~min}$ after the onset of ischemia. To further test whether the cardioprotective effect of esmolol + milrinone was dependent on Akt activation, IS was assessed in a separate group of animals $(n=12)$ who received Akt inhibition with AKT $1 / 2$ kinase inhibitor (Sigma, $2 \mathrm{mg} / \mathrm{kg}$, infused at $10 \mathrm{~min}$ after the onset of ischemia) followed by administration of esmolol + milrinone. The effect of AKT 1/2 kinase inhibitor on IS was also tested separately $(n=8)$.

Effect of esmolol + milrinone on myocyte protection in vitro To confirm the in vivo intra-ischemic myocyteprotection of esmolol + milrinone, we studied their effects in a well validated adult rat mixed cardiomyocyte culture preparation [5, 6]. After $24 \mathrm{hr}$ culture, hypoxia was induced by layering mineral oil over media covering the cells for $2 \mathrm{hr}$ followed by $60 \mathrm{~min}$ re-oxygenation. Saline, esmolol $(1 \mathrm{nmol} / \mathrm{L})$, milrinone $(4.7 \mu \mathrm{mol} / \mathrm{L})$ or esmolol + milrinone was individually applied to the myocyte culture at the $30 \mathrm{~min}$ after the onset of ischemia. The effects of esmolol + 
milrinone on myocyte survival were also tested in the presence of Rp-cAMPS $(10 \mu \mathrm{mol} / \mathrm{L})$ or AKT $1 / 2$ kinase inhibitor $(10 \mu \mathrm{mol} / \mathrm{L})$. Myocyte death rates were quantified by counting trypan blue-stained cells and expressed as a percentage of the total cells counted ( $n=8 /$ group). The examiner who counted cells was blinded to the treatment assignments.

To determine whether Rp-cAMPS exerts any non-specific effects on ventricular myocyte, we tested the effect of RpcAMPS on isolated ventricular myocytes depleted with ICA cells. This is an important issue, since in the intact heart or cultured cardiocyte mixture, there are endogenous and constitutive adrenopeptidergic neuroparacrine-tomyocyte inputs (derived from ICA cells) via cAMP/PKAdependent signaling [4-6]. Removal of ICA cells from myocytes allows direct assessment of the effect of RpcAMPS on myocytes without introducing confounding effect. In ICA cell-free myocyte preparation, we further tested whether Rp-cAMPS specifically blocked the effect of known PKA activators (ie, $\beta_{2}$-AR agonist terbutaline plus CGRP) $[6,10]$. The ICA cell-free myocytes were prepared by mechanical depletion of ICA cells from dissociated rat ventricular cardiocytes using magnetic purification method [4-6]. After 24-hr cell culture, saline, Rp-cAMPS $(100 \mu \mathrm{mol} / \mathrm{L})$, or the combination of CGRP $(5 \mu \mathrm{mol} / \mathrm{L})$ and $\beta_{2}$-AR agonist terbutaline $(10 \mathrm{nmol} / \mathrm{L})$ were applied individually to ICA cell-free myocyte culture at $30 \mathrm{~min}$ after the onset of hypoxia. In addition, the effect of terbutaline + CGRP was tested in the presence of RpcAMPS applied 10-min before the administration of the drugs. Myocyte death rate was quantified by counting trypan blue-stained cells and expressed as a percentage of the total cells counted ( $n=6 /$ group).

Effect of esmolol + milrinone on myocardial PKA and Akt In the same ischemia model, saline, esmolol, milrinone or esmolol + milrinone were IV infused over 10-min (5 min before and after the onset of reperfusion, $n=6 /$ group). Ten minutes into the reperfusion, LV tissues just below the ligation snare were removed and stored in liquid nitrogen for PKA and Akt assay. To standardize tissue preparation, the anterior half of LV myocardial tissue $(3 \mathrm{~mm}$ below the ligation) extending to the apex was removed for the analysis. Tissue PKA activity was determined using a SignaTECT cAMP-dependent PKA assay system (Promega, Madison, WI). Western blot was performed to detect Akt activity (Ser 473). Dilution for anti-pAkt and anti-Akt (Santa Cruz Biotechnology, Santa Cruz, CA) was 1:1000 [15]. To determine whether the Akt activation is dependent on PKA signaling pathway, animals $(n=4)$ were pretreated with Rp-cAMPS ( $2 \mathrm{mg} / \mathrm{kg} \mathrm{IV}$ ) $10 \mathrm{~min}$ after the onset of ischemia followed by the administration of esmolol + milrinone. Then, myocardial pAkt activity was determined as described above.

TUNEL staining Immunoflurosecent and terminal dUTP nick-end labeling (TUNEL) were performed in 4 groups of ischemic/reperfused rat hearts treated with saline, esmolol, milrinone or esmolol + milrinone. The methods for tissue preparation and TUNEL staining were described elsewhere [6]. Tissues were harvested at $4 \mathrm{hr}$ after reperfusion for apoptosis determination. Adult rat cardiac myocyte is binucleated [19]. It is possible that only one of nuclei of myocyte exhibits TUNEL-positive staining when it undergoes apoptosis at early stage. Since one (not necessarily two) TUNEL-positive nuclei is required to define an apoptotic myocyte, the \% apoptotic cells/section was estimated by counting the number of TUNEL-staining nuclei divided by $1 / 2$ total number of propidium iodidepositive nuclei in 10 randomly selected fields ( $n=6$ slides/ heart). Six hearts for each treatment group were studied. To confirm that apoptotic nuclei are truly belongs to true ventricular myocytes, additional TUNEL staining was performed in conjunction with immunohistochemical colocalization of myocyte-specific marker $\alpha$-actinin using DeadEnd Fluorometric TUNEL System Kit (Promega, Madison, WI). Mouse anti- $\alpha$-actinin (1:100 dilution, Abcam Cambridge MA,) was used as the primary antibody to counter label myocytes. Secondary antibody was Alexa Fluor 568 goat anti-mouse $\operatorname{IgG}(\mathrm{H}+\mathrm{L})(1: 1000$ dilution, Invitrogen Corporation, Carlsbad, CA). TUNEL-positive nuclei within counter-stained myocytes were analyzed from control, esmolol, milrinone and esmolol + milrinone treated groups. Ten randomly selected fields were analyzed for each tissue section and six slides were countered for each heart ( $n=6$ hearts/group). The number of apoptotic myocytes in control group was compared to esmolol, milrinone, and esmolol + milrinone treated group.

Data analysis Two-way ANOVA (saline vs. milrinone in the absence and presence of esmolol) was used for analyzing LV-IS, PKA and Akt activation and apoptosis in ischemia/reperfusion studies with the Bonferroni adjustment for intra-group comparisons. All data are presented as mean \pm SD. The significance level $\alpha$ is 0.05 .

\section{Results}

Effect of esmolol + milrinone on LV-IS and myocyte protection Compared with saline control, 13\%, 45\% and $65 \%$ IS-reduction were observed in animals receiving reperfusion treatment of esmolol, milrinone or esmolol + milrinone, respectively ( $n=12$ /group, $p<0.01$, Fig. 1$)$. IS- 

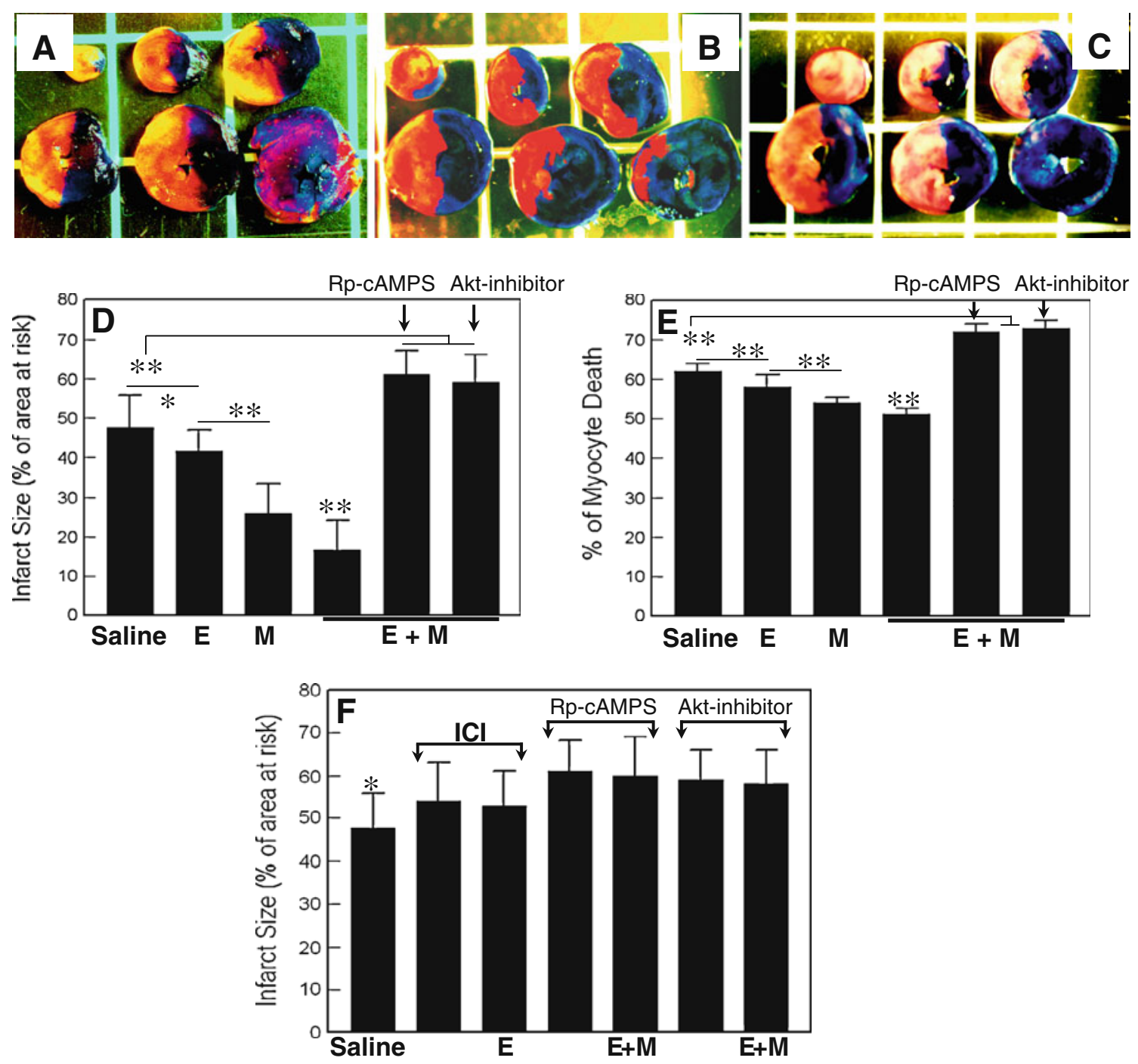

Fig. 1 Panels $\mathrm{A}, \mathrm{B}$ and $\mathrm{C}$ show representative cases of IS in the animals treated with saline (a), esmolol + milrinone $(E+M)(b)$ and $\mathrm{E}+\mathrm{M}$ in the presence of Rp-cAMPS (c). Infarcted tissue is indicated with yellow color. Panel $\mathbf{d}$ shows the IS affected by the therapy with esmolol, milrinone or $\mathrm{E}+\mathrm{M}$ in the absence and presence of $\mathrm{Rp}$ cAMPS or AKT $1 \frac{2}{2}$ kinase inhibitor ( $n=12 /$ group). IS in $\mathrm{E}+\mathrm{M}$-treated group is significantly smaller compared with all other groups $\left(^{*}: p<\right.$ $0.05, * *: p<0.01)$. Panel E: the effects of intra-ischemic application of esmolol, milrinone or $\mathrm{E}+\mathrm{M}$ in the absence and presence of $\mathrm{Rp}$ -

reduction conferred by esmolol was abolished in the animals pretreated with the $\beta_{2}$-AR antagonist ICI-118,551 which alone increased IS $(p<0.05 n=8)$, compared with saline (Fig. 1f). Esmolol + milrinone conferred 35\% and $59 \%$ more IS reduction compared with milrinone or esmolol alone, respectively $(p<0.05)$. In the presence of Rp-cAMPS or AKT $1 / 2$ kinase inhibitor, esmolol + milrinone conferred no reduction on IS. Rp-cAMPS or Akt inhibitor alone increased IS, compared with saline group ( $p<0.05, n=8$, Fig. 1f). There were no significant differences in animal weight and area-at-risk among all the groups (data not shown). There was no significant change
cAMPS or AKT $1 / 2$ kinase inhibitor on myocyte death rates during hypoxia/re- $\mathrm{O}_{2}$ in myocyte culture preparation ( $n=8 /$ group). Panel $\mathbf{f}$ : effects of $\beta_{2}$-AR antagonist ICI-118,551 (ICI), Rp-cAMPS, or AKTinhibitor alone on IS, comparing with saline ( $n=8 /$ group). Effects of esmolol, milrinone and esmolol + esmolol in the presence of their respective antagonists were plotted for comparison. Myocyte death rate in $\mathrm{E}+\mathrm{M}$ treated group is significantly lower compared with all other groups $(*: p<0.05, * *: p<0.01)$

in arterial BP following esmolol + milrinone infusion. A $6 \% \mathrm{HR}$ reduction was observed at 6 min following the completion of esmolol + milrinone infusion (Tables 1, 2, 3 ). There was no post-ischemic mortality in the animals receiving esmolol, milrinone or esmolol + miltinone. The mortality rates were $10 \%, 30 \%$ and $35 \%$ in saline, Rp-cAMPS, and Akt-inhibitor groups, respectively. In mixed myocyte cultures, treatment with esmolol, milrinone and esmolol + milrinone reduced cell death rates by $5.5 \%, 13.3 \%$ and $16.8 \%$, respectively, compared with saline ( $n=8 /$ group, $p<0.01$, Fig. 1 ). The myocyte death rates of esmolol + milrinone group in the presence of 
Table 1 Hemodynamic changes following intravenous infusion of esmolol + milrinone

\begin{tabular}{lccc}
\hline $\begin{array}{l}\text { Esmolol }+ \\
\text { Milrinone }\end{array}$ & $\begin{array}{l}\text { 25-min into } \\
\text { ischemia (5-min } \\
\text { before reperfusion) }\end{array}$ & $\begin{array}{l}\text { 10-min after } \\
\text { completion } \\
\text { of esmolol }+ \\
\text { milrinone infusion }\end{array}$ & $\mathrm{P}$ \\
\hline HR & $241 \pm 29$ & $227 \pm 16$ & $<0.01$ \\
Systolic BP & $140 \pm 16$ & $135 \pm 13$ & $\mathrm{NS}$ \\
Diastolic BP & $120 \pm 5$ & $110 \pm 6$ & $\mathrm{NS}$ \\
HR $\times$ BP & $33636 \pm 2669$ & $30661 \pm 1675$ & $<0.01$ \\
\hline
\end{tabular}

Rp-cAMPS or Akt inhibitor were higher than that of saline control (Fig. 1). In ICA cell-free myocyte preparation, the effect of Rp-cAMPS on myocytes was not different from that of saline during hypoxia/re-oxygenation $(p=\mathrm{ns}, n=6$, Fig. 2). The myocyte protective effect conferred by terbutaline + CGRP was abolished in the presence of RpcAMPS (Fig. 2).

\section{Effect of esmolol + milrinone on myocardial PKA and Akt} activation Five min after completing infusion of esmolol, milrinone or esmolol + milrinone, there was $22 \%, 28 \%$ and $59 \%$ increase in ischemic LV tissue PKA activity, respectively, compared with control (Fig. 3). In parallel with PKA changes, esmolol, milrinone and esmolol + milrinone treatment increased pAkt levels in ischemic LV tissue by 1.7-, 2.7-, and 6-fold, respectively. There was no increase in cardiac pAkt activity in those animals pretreated with RpcAMPS. Compared with saline treatment, Rp-cAMPS or esmolol + milrinone in the presence of Rp-cAMPS induced 0.8- and 0.9- fold pAkt changes ( $p=n s$, Fig. 4 ).

Effect of esmolol + milrinone on myocyte apoptosis Early reperfusion treatment of LV myocardium with esmolol, milrinone or esmolol + milrinone reduced overall cardiocyte apoptosis by $23 \%, 37 \%$ and $60 \%$, respectively, compared with saline control (Fig. 5). Myocyte-specific TUNE staining demonstrated $28 \%, 40 \%$ and $67 \%$ reduction in apoptosis in esmolol, milrinone and esmolol + milrinone

Table 2 Hemodynamic changes following intravenous infusion of esmolol

\begin{tabular}{lccc}
\hline Esmolol & $\begin{array}{l}\text { 25-min into } \\
\text { ischemia (5-min } \\
\text { before reperfusion) }\end{array}$ & $\begin{array}{l}\text { 10-min after } \\
\text { completion of } \\
\text { esmolol infusion }\end{array}$ & $\mathrm{P}$ \\
\hline HR & $240 \pm 28$ & $218 \pm 16$ & $<0.01$ \\
Systolic BP & $142 \pm 16$ & $140 \pm 13$ & $\mathrm{NS}$ \\
Diastolic BP & $120 \pm 8$ & $116 \pm 6$ & $\mathrm{NS}$ \\
HR x BP & $34080 \pm 2568$ & $28340 \pm 1675$ & $<0.01$ \\
\hline
\end{tabular}

Table 3 Hemodynamic changes following intravenous infusion of milrinone

\begin{tabular}{lccc}
\hline Milrinone & $\begin{array}{l}\text { 25-min into } \\
\text { ischemia (5-min } \\
\text { before reperfusion) }\end{array}$ & $\begin{array}{l}\text { 10-min after } \\
\text { completion of } \\
\text { milrinone infusion }\end{array}$ & P \\
\hline HR & $241 \pm 29$ & $246 \pm 28$ & $<0.05$ \\
Systolic BP & $141 \pm 16$ & $137 \pm 13$ & NS \\
Diastolic BP & $120 \pm 5$ & $115 \pm 6$ & NS \\
HR $\times$ BP & $33980 \pm 2615$ & $33702 \pm 2642$ & NS \\
\hline
\end{tabular}

treatment group, respectively, compared with saline control (Fig. 5).

\section{Discussion}

We have demonstrated for the first time that brief IV infusion of esmolol + milrinone at late-ischemia and early-reperfusion (i.e., bridging from end of coronary occlusion to re-canalization) confers robust additive LVIS-reduction. This cardioprotective effect is associated with the activation of PKA/Akt-dependent antiapoptotic signaling.

Effect of esmolol + milrinone on LV-IS Ischemic preconditioning with milrinone reduces LV-IS in a canine reperfused AMI model [14]. However, until proven otherwise, the pharmacological ischemic preconditioning cannot generalize the same mechanism and outcome when the same agent is administered in ischemic postconditioning setting. The cardioprotective effect of intra-ischemic milrinone administration has not been fully established. The cellular mechanisms underlying milrinone-mediated ischemic postconditioning are unknown. Inconsistent results have been published with respect to the effects of intra-ischemic administration of milrinone on the heart $[17,20]$. Intra-ischemic milrinone infusion in a non-reperfused canine AMI model failed to reduce LV-IS [20]. In contrast, a recent study reported that intra-ischemic milrinone infusion reduced right ventricular IS in a canine ischemia-reperfusion model [17], although the latter study provided no data on the effect of milrinone on LVIS [17]. The lack of milrinone-induced LV-IS-reduction in non-reperfused AMI [20] could be explained by the lack of reperfusion injury, a process that would have been effectively targeted by milrinone. In an isolated perfused rabbit heart model, milrinone had no effect on LV-IS [21]. This could be due to the fact that isolated hearts already underwent a brief episode of ischemia preconditioning during the heart isolation procedure thereby blunting subsequent milrinone's effect. The 


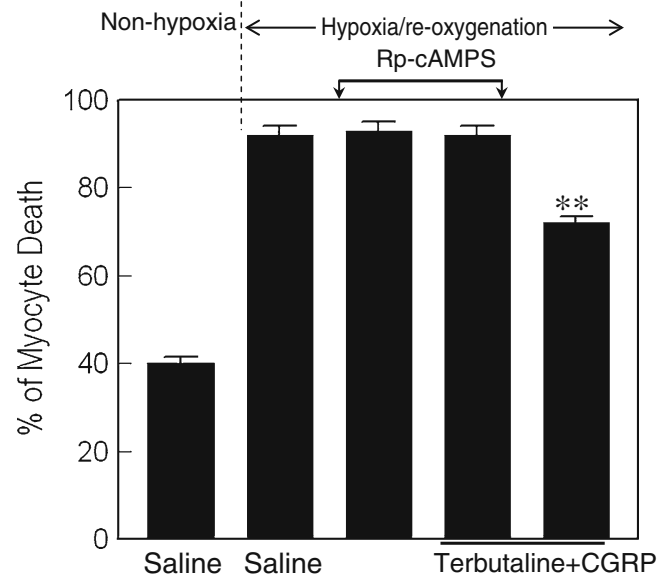

Fig. 2 Effect of Rp-cAMPS on myocyte survival during hypoxia/reoxygenation in ICA cell-free ventricular myocyte culture. There is no difference in myocyte survival rates between saline and Rp-cAMPS groups ( $\mathrm{p}=\mathrm{ns}, n=6 /$ group). Rp-cAMPS abolishes myocyte protective effect conferred by PKA agonists (Terbutaline + CGRP). Hypoxia/reoxygenation markedly increases myocyte death rates compared with non-hypoxic condition in ICA cell-free myocyte culture. ${ }^{* *}: p<0.01$

present study is the first to show that late-ischemia/early reperfusion administration of milrinone reduced LV-IS. Furthermore, we demonstrated that early reperfusion administration of esmolol + milrinone conferred additive LV-ISreduction. The cell protective effect conferred by esmolol + milrinone was confirmed in an isolated mixed myocyte preparation in the present study. Endogenous epinephrine derived from ICA cells confers significant myocardial protection against reperfusion injury through $\beta_{2}$-AR stimulation $[5,6]$. In consistent with this concept, the present study demonstrated that esmolol's IS-reduction is primarily mediated via the enhancement of cardiac $\beta_{2}$-AR signaling. It has been demonstrated that myocardial interstitial epinephrine levels can increase by $>100$ fold in the early coronary

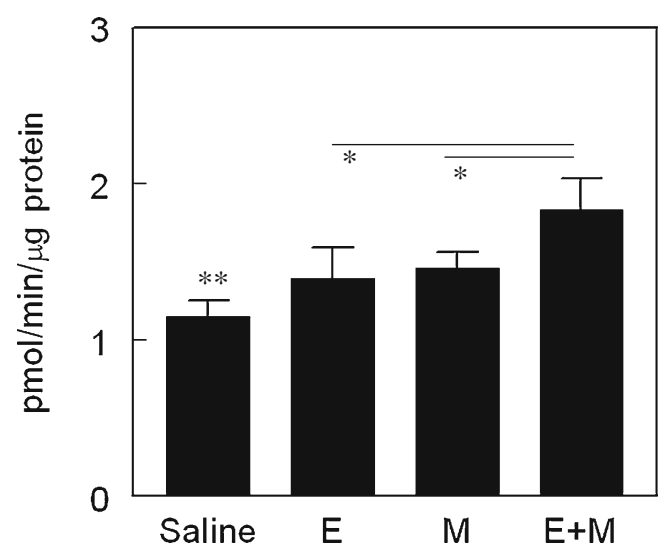

Fig. 3 Effects of reperfusion therapy with esmolol, milrinone or E + M on LV myocardial tissue PKA during ischemia/reperfusion $(n=6 /$ group). PKA activity in saline treated group is significantly lower than all other groups. *: $p<0.05, * *: p<0.01$ reperfusion [16]. Thus, $\beta_{1}$-AR blockade with esmolol during early reperfusion can exert unopposed $\beta_{2}$-AR stimulation via endogenous epinephrine producing myocardial protection. Reduction in myocardial oxygen consumption by esmolol during ischemia can potentially contribute to LV-IS-reduction. However, cardioprotection conferred by esmolol + milrinone in the present study is unlikely due to its favorable hemodynamic profile as reflected by the low BP-HR double product, since esmolol alone which had comparable hemodynamic effect only produced modest LV-IS-reduction. The IS-reduction conferred by esmolol + milrinone can be reasonably attributed to their effects against reperfusion injury, since the drugs were given towards the very end of ischemia and the beginning of reperfusion.

Administration of Rp-cAMPS or Akt-inhibitor increases IS, compared with saline control. These data indicate that there is a basal tonic PKA-Akt signaling during ischemia/ reperfusion in the heart. Disrupting this signaling pathway can result in catastrophic reperfusion injury. In the presence of Rp-cAMPS or Akt-inhibitor, esmolol/milrinone conferred no IS-reduction. This finding indicates that mechanism of esmolol/milrinone-induced heart protection is mediated through the PKA/Akt signaling cascade. The cardiac effects of Rp-cAMPS are unlikely to be nonspecific in the present study. Our data (Fig. 2) indicates that Rp-cAMPS, in the absence of background extracellular ligand-mediated PKA stimulation, acts no more than saline on purified myocytes (depleted with ICA cells). In ICA cell-free myocyte preparation, Rp-cAMPS abolished myocyte protective effect conferred by terbutaline + CGRP, the agonists of $\beta_{2}$-AR/CGRP-receptors known to stimulate PKA with IS reduction $[6,10]$. These findings confirmed the specificity of Rp-cAMPS on PKA's inhibition and confirmed in vivo data that cardioprotection conferred by milrinone + esmolol is mediated specifically through the activation of cAMP-PKA dependent signaling pathway.

There are limited clinical therapies to reduce reperfusion injury in AMI [22]. Recent experiments demonstrated that cardiac PKA activation using concomitant $\beta_{2}$-AR/CGRPreceptor stimulation with the receptor agonists (i.e., terbutaline and CGRP) at early reperfusion conferred additive cardioprotection with a 74\% LV-IS-reduction compared with saline [10]. The present study investigates a more distal signaling step (i.e., skipping $\beta_{2}$-AR/CGRP-receptor$G$ protein-coupling sequence) to directly activate downstream PKA. This strategy allows the most rapid PKA activation at the earliest reperfusion time. Milrinone is known to activate PKA in non-ischemic [13] and ischemic myocardium [14]. Esmolol may indirectly activate PKA at early reperfusion through enhanced endogenous $\beta_{2}$-AR stimulation. Esmolol + milrinone infusion at 5-min before the onset of reperfusion offers a "priming" effect of PKA 
A

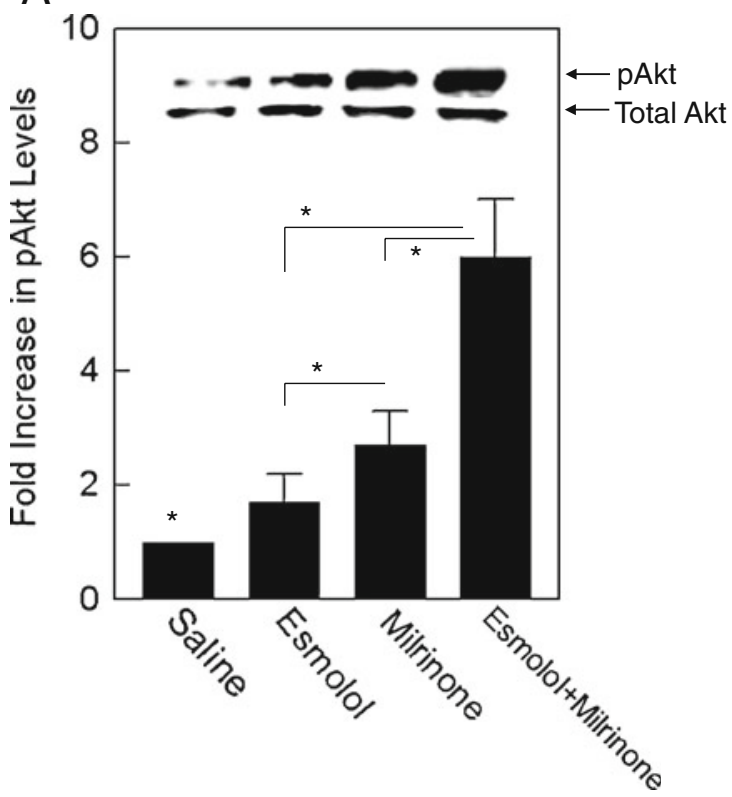

B

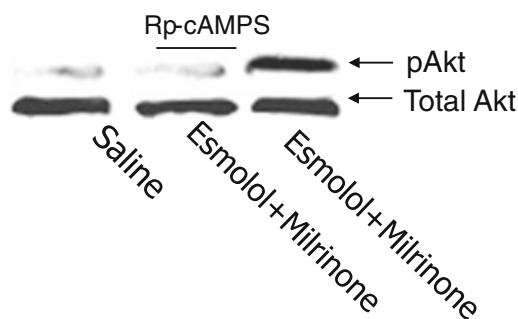

Fig. 4 Panel A: Effects of reperfusion therapy with esmolol, milrinone or $\mathrm{E}+\mathrm{M}$ on myocardial tissue Akt activity during ischemia/reperfusion ( $n=5 /$ group). The pAkt activity in saline treated group is significantly lower compared with all other group $(* *: p<$ $0.01)$. The phosphorylated Akt levels were normalized to total Akt. Insert: an individual case of myocardial tissue pAkt levels in response to different treatments. Panel B: A typical case demonstrating that in the presence of PKA inhibitor Rp-cAMPS, E + M exerts no effect on myocardial pAkt activity. Overall, application of $\mathrm{E}+\mathrm{M}$ increases myocardial pAkt activity by $7 \pm 1$-fold, compared with saline $(p<0.01$, $n=4$ /group). In the presence of Rp-cAMPS, $\mathrm{E}+\mathrm{M}$ has no effect on myocardial tissue pAkt activity during ischemia/reperfusion, compared with saline ( $n=4$ /group)

Akt inhibition suggests that ischemia-reperfusion per se can trigger a small but significant amount of PKA/Aktdependent antiapoptotic signaling which can be readily enhanced pharmacologically during the very early phase of reperfusion.

The $\beta_{1}$-AR blocker is the standard of therapy for patients with AMI to improve long-term survival [24-26]. The mechanisms underlying cardioprotection of $\beta_{1}$-AR blocker may not be simply attributed to its reducing myocardial oxygen consumption or blocking $\beta_{1}$-AR signaling which is detrimental to the heart. It has been well recognized that $\beta_{1}$-AR and $\beta_{2}$-AR signaling are counterregulatory $[11,12]$. Myocardial $\beta_{1}$-AR inhibition greatly enhances cardioprotective $\beta_{2}$-AR signaling which activates antiapoptotic pathway $[11,12]$. Selective $\beta_{1}$-AR blockade with esmolol significantly enhanced milrinone-mediated myocardial protection. These results underscore the importance of intrinsic cardiac epinephrine- $\beta_{2}$-AR signaling derived from ICA cells providing rich myocardial epinephrine $[5,6]$. Furthermore, our findings indicate that activation of $\beta_{2}$-AR in concert with direct PKA activation with milrinone confers powerful heart protection. Accumulating evidence supports a concept that apoptosis contributes substantially to the extent of IS in the reperfused AMI [2]. In agreement with this, the ratios of IS-reduction fact that the larger than control LV-IS following PKA or 
Fig. 5 (a) Effects of reperfusion therapy with esmolol, milrinone or $\mathrm{E}+\mathrm{M}$ on LV myocyte apoptosis during ischemia/ reperfusion. Panels A-C show a case of total cardiocyte apoptosis in a saline-treated heart.

A: Propidium iodide-positive nuclei (red) of cardiocytes. B: TUNEL-positive nuclei (green, arrows) in the same field. C: Superimposed panels A and B. Panels D-F show a case of cardiocyte apoptosis in an $\mathrm{E}+\mathrm{M}$-treated heart.

D: Popidium iodide-positive nuclei of cardiocytes.

E: TUNEL-positive nuclei in the same field of D.

F: Superimposed panels D and

F. Panels

G-H: Immunohistochemical double staining confirms myocyte (red)-specific apoptosis in a field of saline-treated heart (G) and field esmolol + milrinone-treated heart $(\mathrm{H})$. TUNEL positive nuclei are stained with green color. Scale bar $=10 \mu \mathrm{m}$. (b). A: Percentage changes in cardiocyte apoptosis in the hearts treated with saline, esmolol, milrinone and $\mathrm{E}+\mathrm{M}$, respectively ( $n=6 /$ group). The cardiocyte apoptosis rate in hearts treated with $\mathrm{E}+\mathrm{M}$ is smaller than all other groups $(p<0.01)$. B: Comparison of myocyte-specific apoptosis among 4 different treatment groups. The extent of ventricular myocyte apoptosis in esmolol + milrinone group is significantly smaller than all other groups $(p<0.01) . *: p<0.05, * *$ : $p<0.01$

a
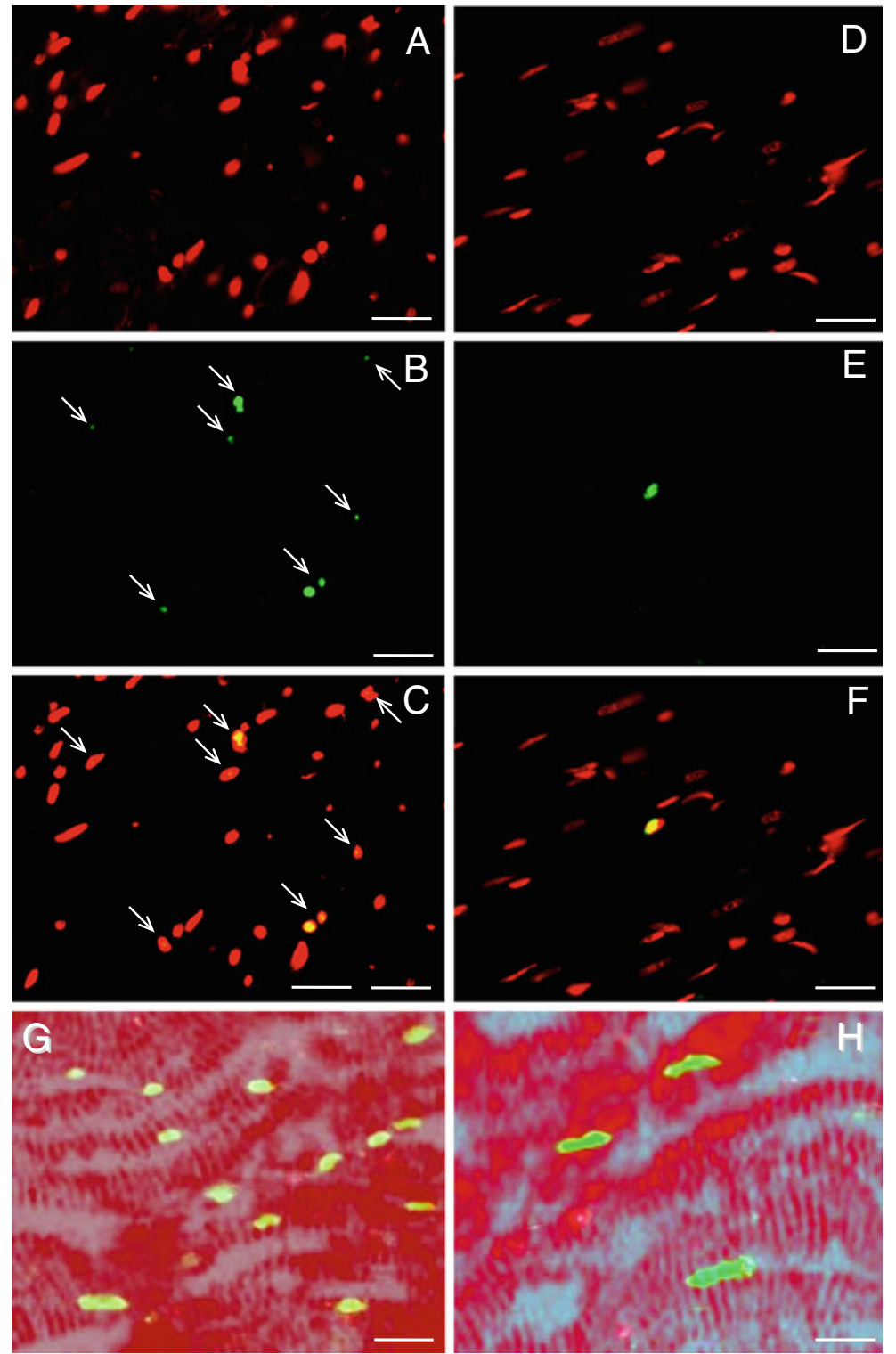

b

\section{A}

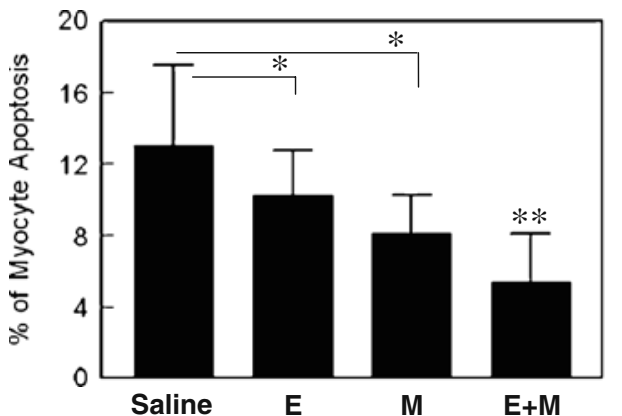

B

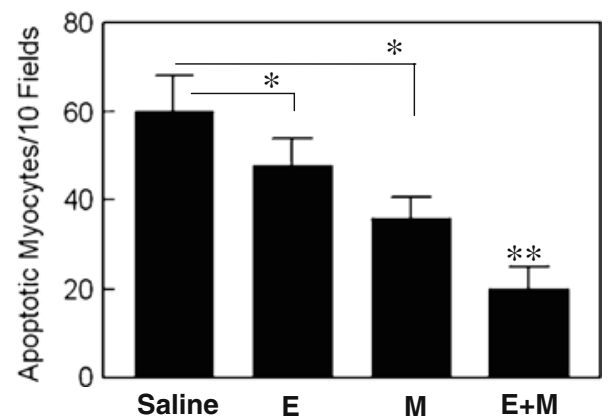


between esmolol + milrinone and other treatment groups are in the similar orders of the rations of myocyte apoptosis reduction in their counterparts. The absolute magnitudes of apoptosis do not mach those of LV-IS. This discrepancy might be due to several reasons. First, the immunostaining might not be sensitive enough to detect all apoptotic nuclei, especially those of early phase. Second, myocyte necrosis certainly contributes to substantial amount of total LV-IS. Nevertheless, the present study provides proof-of-theconcept that anti-apoptotic effect elicited by esmolol + milrinone contributes significantly to the reduced reperfusion injury.

Perspectives There is no clinically approved drug that can effectively reduce reperfusion injury during AMI. For noncardioprotective purpose, esmolol or milrinone was used individually with good tolerance in patients with AMI with or without reperfusion therapy [27, 28]. Although the cardioprotective efficacy of combination therapy with esmolol + milrinone has not been tested clinically, their remarkable preclinical LV-IS-limiting effect, if confirmed in human study, may make this novel combination a promising candidate for transition to the clinical arena.

Study limitation The present study cannot deduce that cardioprotective results of esmolol+milrinone are class effects of $\beta_{1}$-AR blockers and PDE inhibitors. The reason for selecting esmolol vs. other $\beta_{1}$-selective AR blockers is that esmolol possesses ultra short half-life providing favorable hemodynamic profile when combined with milrinone infusion. Further studies are needed to test whether other $\beta_{1}$-AR blockers and PDE inhibitors confer similar cardioprotection. While the PKA phosphorylation was used to determine PKA activity in the present study, we have not assessed other molecular markers associated with PKA activation such as cytosolic cAMP levels or phosphorylation of the L-type calcium channels.

In conclusion, late-ischemia/early reperfusion therapy with combination of esmolol + milrinone confers additive LV-IS-reduction in experimental AMI. This cardioprotective effect is associated with a robust induction of antiapoptotic signaling pathway.

Acknowledgements We thank Dr. Don Deyo for technical support of animal study.

Funding Sources This study was supported by the American Heart Association National Scientist Development Grant (to MH), the Society of Geriatric Cardiology (to $\mathrm{MH}$ ) and the National Institute of Health (NIH HL085487 to BKM).

Conflict of Interest No potential interest conflicts.
Open Access This article is distributed under the terms of the Creative Commons Attribution Noncommercial License which permits any noncommercial use, distribution, and reproduction in any medium, provided the original author(s) and source are credited.

\section{References}

1. Hausenloy DJ, Yellon DM. New directions for protecting the heart against ischemia-reperfusion injury: targeting the reperfusion injury salvage kinase (RISK)-pathway (review). Cardiovasc Res. 2004;61:448-60.

2. Ovitz M, Baxter GF, Di Lisa F, Ferdinandy P, Garcia-Dorado D, Hausenloy D, et al. Postconditioning and protection from reperfusion injury: where do we stand? Cardiovasc Res (Review). 2010;87:406-23.

3. Skyschally A, Gaster PV, Boengler K, Gres P, Musiolik J, Schilawa D, et al. Ischemic postconditioning in pigs no causal role for RISK activation. Circ Res. 2009;104:15-8.

4. Huang MH, Friend DS, Sunday ME, Singh K, Haley K, Austen $\mathrm{KF}$, et al. An intrinsic adrenergic system in mammalian heart. J Clin Invest. 1996;98:1298-303.

5. Huang MH, Wang HQ, Roeske WR, Birnbaum Y, Wu Y, Yang NP, et al. Mediating $\delta$-opioid-initiated heart protection via the $\beta_{2}$-adrenergic receptor: role of the intrinsic cardiac adrenergic cell. Am J Physiol Heart Circ Physiol. 2007;293: H376-84.

6. Huang MH, Nguyen V, Wu Y, Rastogi S, Birnbaum Y, Lui CY, et al. Reducing ischemia/reperfusion injury through $\delta$-opioidregulated intrinsic cardiac adrenergic cells: adrenopeptidergic co-signaling. Cardiovasc Res. 2009;84:452-60.

7. Aiyar N, Rand K, Elshourbagy NA, Zeng Z, Adamou JE, Bergsma DJ, et al. A cDNA encoding the calcitonin gene-related peptide type 1 receptor. J Biol Chem. 1996;271:11325-9.

8. Huang MH, Knight III PR, Izzo JL. $\mathrm{Ca}^{2+}$-dependent $\mathrm{Ca}^{2+}$ mobilization involved in CGRP-mediated positive inotropic effect in ventricular myocytes. Am J Physiol Regul Integr Comp Physiol. 1999;276:R259-64.

9. Tong H, Bernstein ME, Steenbergen C. The role of beta-adrenergic receptor signaling in cardioprotection. FASEB J. 2005;19:983-5.

10. Huang MH, Nguyen V, Rastogi S, Wu Y, Birnbaum Y, Lin Y, et al. Post-ischemic therapy with calcitonin gene-related peptide and $\beta_{2}$-adrenergic receptor agonist confers synergistic infarct size reduction. Circ Res (abstract). 2007;101:1210.

11. Communal C, Singh K, Sawyer DB, Colucci WS. Opposing effects of $\beta_{1^{-}}$and $\beta_{2}$-adrenergic receptors on cardiac myocyte apoptosis: role of a pertussis toxin-sensitive $\mathrm{G}$ protein. Circulation. 1999;100:2210-2.

12. Zhu WZ, Zheng M, Koch WJ, Lefkowitz RJ, Kobilka BK, Xiao RP. Dual modulation of cell survival and cell death by $\beta_{2}$ adrenergic signaling in adult mouse cardiac myocytes. Proc Natl Acad Sci USA. 2001;98:1607-12.

13. Haikala H, Kaheinen P, Levijoki, Linden IB. The role of cAMP- and cGMP-dependent protein kinases in the cardiac actions of new calcium sensitizer Levosimendan. Cardiovasc Res. 1997;34:536-46.

14. Sanada S, Kitakaze M, Papst PJ, Asanuma H, Node K, Takashima $\mathrm{S}$, et al. Cardioprotective effect afforded by transient exposure to phosphodiesterase III inhibitor: the role of protein kinases A and p38 mitogen-activated protein kinase. Circulation. 2001;104:70510.

15. Geissler HJ. Reduction of myocardial reperfusion injury by highdose beta-blocker with esmolol. Thorac Cardiovasc Surg. 2002;50:367-72.

16. Killingsworth CR, Wei C-C, Dell'Italia LJ, Ardell JL, Kingsley MA, Smith WM, et al. Short-acting $\beta$-adrenergic antagonist 
esmolol given at reperfusion improves survival after prolonged ventricular fibrillation. Circulation. 2004;109:2469-74.

17. Hein M, Roehl AB, Baumert JH, Scherer K, Steendijk P, Rossaint R. Anti-ischemic effects of inotropic agents in experimental right ventricular infarction. Acta Anaesthesiol Scand. 2009;53:941-8.

18. Wu Y, Yin X, Wijaya C, Huang M, McConnell BK. Acute myocardial infarction in rats. JoVE. 2011, 48: http://www.jove. com/index/details.stp?ID=2464. doi: 10.3791/2464.

19. Katzberg AA, Farmer BB, Harris RA. The predominance of binucleation in isolated rat heart myocytes. Am J Anat. 2005;149:489-99.

20. Campbell CA, Reddy BR, Alker KJ, Wynne J, Kloner RA. Effect of milrinone on acute myocardial infarct size. Am J Cardiol. 1987;60:422-3.

21. Fukasawa M, Nishida H, Sato T, Miyazaki M, Nakaya H. 6-[4(1-Cyclohexyl-1H-tetrazol-5-yl)butoxy]-3,4-dihydro-2-(1H)quinolinone (cilostazol), a phosphodiesterase type 3 inhibitor, reduces infarct size via activation of mitochondrial $\mathrm{Ca}^{+}$activated $\mathrm{K}^{+}$channels in rabbit hearts. J Pharmacol Exp Ther. 2008;326:100-4.

22. Piot C, Croisille P, Staat P, Thibault H, Rioufol G, Mewton N, et al. Effect of cyclosporine on reperfusion injury in acute myocardial infarction. N Engl J Med. 2008;359:473-81.
23. Morisco C, Condorelli G, Trimarco V, Bellis A, Marrone C, Sadoshima J, et al. Akt mediates the cross-talk between betaadrenergic and insulin receptors in neonatal cardiomyocytes. Circ Res. 2005;96:180-8.

24. Halkin A, Grines CL, Cox DA, Garcia E, Mehran R, Tcheng JE, et al. Impact of intravenous beta-blockade before primary angioplasty on survival in patients undergoing mechanical reperfusion therapy for acute myocardial infarction. J Am Coll Cardiol. 2004;43:1780-7.

25. Pedersen TR. The Norwegian multicenter study of timolol after myocardial infarction. Circulation. 1983;67:I49-53.

26. Gottlieb SS, McCarter RJ, Vogel RA. Effect of beta-blockade on mortality among high-risk and low-risk patients after myocardial infarction. N Engl J Med. 1998;339:489-97.

27. Karlsberg RP, DeWood MA, DeMaria AN, Berk MR, Lasher KP. Comparative efficacy of short-term intravenous infusions of milrinone and dobutamine in acute congestive heart failure following acute myocardial infarction. Milrinone-Dobutamine Study Group. Clin Cardiol. 1996;19:21-30.

28. Mooss AN, Hilleman DE, Mohiuddin SM, Hunter CB. Safety of esmolol in patients with acute myocardial infarction treated with thrombolytic therapy who had relative contraindications to betablocker therapy. Ann Pharmacother. 1994;28:701-3. 